\title{
NGC 146: a young open cluster with a Herbig Be star and intermediate mass pre-main sequence stars
}

\author{
A. Subramaniam ${ }^{1}$, D. K. Sahu ${ }^{2}$, R. Sagar ${ }^{3}$, and P. Vijitha ${ }^{4}$ \\ ${ }^{1}$ Indian Institute of Astrophysics, II Block Koramangala, Bangalore 560034, India \\ e-mail: purni@iiap.res.in \\ 2 CREST Campus, Indian Institute of Astrophysics, Hosakote, Bangalore, India \\ e-mail: dks@crest.ernet.in \\ 3 Aryabhatta Research Institute of Observational Studies (ARIES), Manora peak, Nainital 263129, India \\ e-mail: sagar@upso.ernet.in \\ 4 VSRTP Student, Mother Theresa University, Kodaikanal, India
}

Received 12 January 2005 / Accepted 11 May 2005

\begin{abstract}
We present $U B V C C D$ photometry and low-resolution spectra of stars in the field of the young open cluster NGC 146. $U B V$ photometry of 434 stars were used to estimate the $E(B-V)$ reddening of $0.55 \pm 0.04$ mag and $B V$ photometry of 976 stars were used to estimate a distance modulus of $(m-M)_{0}=12.7 \pm 0.2 \mathrm{mag}$, corresponding to a distance of $3470_{-305}^{+335} \mathrm{pc}$. We estimated 10-16 Myr as the turn-off age for the upper main sequence of the cluster using isochrones and synthetic colour magnitude diagrams. We identified two $\mathrm{B}$ type stars with $\mathrm{H}_{\alpha}$ in emission and located on the MS using slit-less spectra. A higher resolution spectrum of the brighter Be star indicated the presence of a number of emission lines, with some lines showing the signature of gas infall. This star was found to be located in the region of Herbig Ae/Be stars in the $(J-H)$ vs. $(H-K)$ colour-colour diagram. Thus, we identify this star as a Herbig Be star. On the other hand, 54 stars were found to show near infrared excess, of which 17 were found to be located in the region of Herbig Ae/Be stars and 18 stars were found to be located in the region of Be stars in the NIR colour-colour diagram. Thus NGC 146 is a young cluster with a large number of intermediate mass pre-main sequence stars. The turn-on age of the cluster is found to be $\sim 3$ Myr. Though NGC 146 shows an older turn off, the bulk of stars in this cluster seems to belong to the younger population of $3 \mathrm{Myr}$.
\end{abstract}

Key words. Galaxy: open cluster and associations: individual: NGC 146 - stars: emission-line, Be stars: Hertzsprung-Russell (HR) and C-M diagrams - stars: pre-main sequence

\section{Introduction}

The open star cluster system of our Galaxy is an important constituent of the disk of the Galaxy. The open star clusters are formed from molecular clouds at the sites of star formation. NGC 146 is a young open cluster located in the direction of the Perseus spiral arm. NGC 146 (RA = 0h 33.05m; Dec $=+6318.1(\mathrm{~J} 2000))$ is located away from the center of the Galaxy $(l=120.9 ; b=0.5)$. The approximate age of the cluster was estimated as $10 \mathrm{Myr}$ and the distance to the cluster was found to be more than $3 \mathrm{kpc}$, which reinforces the assertion that there may be a spiral arm beyond the Perseus arm (Phelps \& Janes 1994). In the present study, we have obtained $U B V$ photometry of the stars in the field of NGC 146 up to a limiting magnitude of $V \sim 20 \mathrm{mag}$. This has helped in estimating the differential reddening across the field of the cluster, thereby obtaining better estimates of the cluster parameters. The cluster has a number of early B type main-sequence stars indicating its young nature, but there are no super giants. The absence of evolved stars has hampered the estimation of age of the cluster using the isochrone fitting method. In this study, we estimate the age of the cluster using the upper main-sequence (MS) as well as pre-main sequence (PMS) stars.

This cluster has been studied previously by Hardorp (1960) using RGU photometry and Jasevicius (1964) using $U B V$ photographic photometry. Phelps \& Janes (1994) obtained CCD photometry of this cluster in $U B V$ passbands. The limit of the photometry was $V \sim 18.0 \mathrm{mag}$. They found the presence of variable reddening across the field of the cluster. The mean reddening was found to be $E(B-V)=0.70$ mag. They estimated a distance of $4786 \mathrm{pc}$ and found that the cluster is younger than $10 \mathrm{Myr}$.

\section{Observation and data analysis}

The cluster was observed using the $2.0 \mathrm{~m}$ Himalayan Chandra Telescope using the HFOSC instrument, in the imaging mode, on 3 July 2003. The CCD used for imaging is a $2 \mathrm{~K} \times 4 \mathrm{~K} \mathrm{CCD}$, where the central $2 \mathrm{~K} \times 2 \mathrm{~K}$ pixels were used. The pixel size is $15 \mu$ with an image scale of $0.297 \mathrm{arcsec} / \mathrm{pixel}$. The total 
Table 1. Journal of observation.

\begin{tabular}{|c|c|c|c|c|}
\hline Date & Filter & $\overline{\mathrm{UT}}$ & Airmass & Exp. Time (s) \\
\hline \multicolumn{5}{|l|}{ Imaging } \\
\hline July 32003 & $V$ & 2122 & 1.36 & 10.0 \\
\hline July 32003 & $V$ & 2126 & 1.35 & 60.0 \\
\hline July 32003 & $V$ & 2130 & 1.34 & 60.0 \\
\hline July 32003 & $V$ & 2134 & 1.33 & 60.0 \\
\hline July 32003 & $V$ & 2140 & 1.32 & 60.0 \\
\hline July 32003 & $U$ & 2154 & 1.29 & 120.0 \\
\hline July 32003 & $U$ & 2159 & 1.28 & 120.0 \\
\hline July 32003 & $B$ & 2213 & 1.26 & 300.0 \\
\hline July 32003 & $B$ & 2221 & 1.25 & 300.0 \\
\hline July 32003 & $B$ & 2230 & 1.24 & 90.0 \\
\hline \multicolumn{5}{|l|}{ Slit-less spectra } \\
\hline 27 January 2004 & $R+$ Grism 5 & & - & 60.0 \\
\hline 27 January 2004 & $R+$ Grism 5 & & - & 600.0 \\
\hline 25 June 2004 & $R+$ Grism 5 & & - & 900.0 ( 2 exposures $)$ \\
\hline 21 July 2004 & $R+$ Grism 5 & & - & 900.0 ( 2 exposures $)$ \\
\hline 23 November 2004 & $R+$ Grism 5 & & - & 900.0 ( 1 exposure $)$ \\
\hline \multicolumn{5}{|l|}{ Slit spectra (S1) } \\
\hline 18 January 2005 & Gr7 & & - & $900.0(3500-7000 \AA)$ \\
\hline 18 January 2005 & Gr8 & & - & $600.0(5200-9200 \AA)$ \\
\hline \multicolumn{5}{|l|}{ Slit spectra (star 1) } \\
\hline 18 January 2005 & Gr7 & & - & $600.0(3500-7000 \AA)$ \\
\hline 18 January 2005 & Gr8 & & - & $600.0(5200-9200 \AA)$ \\
\hline
\end{tabular}

area observed is approximately $10 \times 10 \operatorname{arcmin}^{2}$. The log of the observations is tabulated in Table 1.

The night of observation was not photometric and hence we used 18 bright stars observed by Phelps \& Janes (1994) as calibration stars. The CCD data were calibrated using IRAF data reduction package. The instrumental magnitudes were estimated using the DAOPHOT II package. The zero point errors are of the order of $0.03 \mathrm{mag}$. The $V$ magnitude and $(B-V)$, $(U-B)$ colours were estimated for 483 stars, whereas only $V$ magnitude and $(B-V)$ colour were estimated for 976 stars. The observed field of the cluster with 976 stars is shown in Fig. 1. From the above data, only stars with errors less than $0.1 \mathrm{mag}$ are used for the estimation of cluster parameters. The cluster was also observed in the slit-less mode with the grism as the dispersing element using the HFOSC. This mode of observation using the HFOSC yields an image where the stars are replaced by their spectra. This is similar to objective prism spectra. The broad band $R$ filter $(7100 \AA, B W=2200 \AA)$ and Grism 5 (5200-10300 $\AA$ ) of HFOSC CCD system was used in combination without any slit. The resolution of grism 5 is 870 . We used the Johnsons $R$ filter in the field to restrict the spectra to the spectral region of the $R$ band. The slit-less spectra thus obtained were used to identify the presence of emission line stars in the field of the cluster. Since NGC 146 is known to be a young cluster with a number of stars populating the $B$-spectral range, there is a good chance of some of the above stars showing emission line features. The first two slit-less spectra were obtained on 27 January 2004. We first obtained an image of the cluster in $R$ filter, followed by 1 and 10 min exposures with the

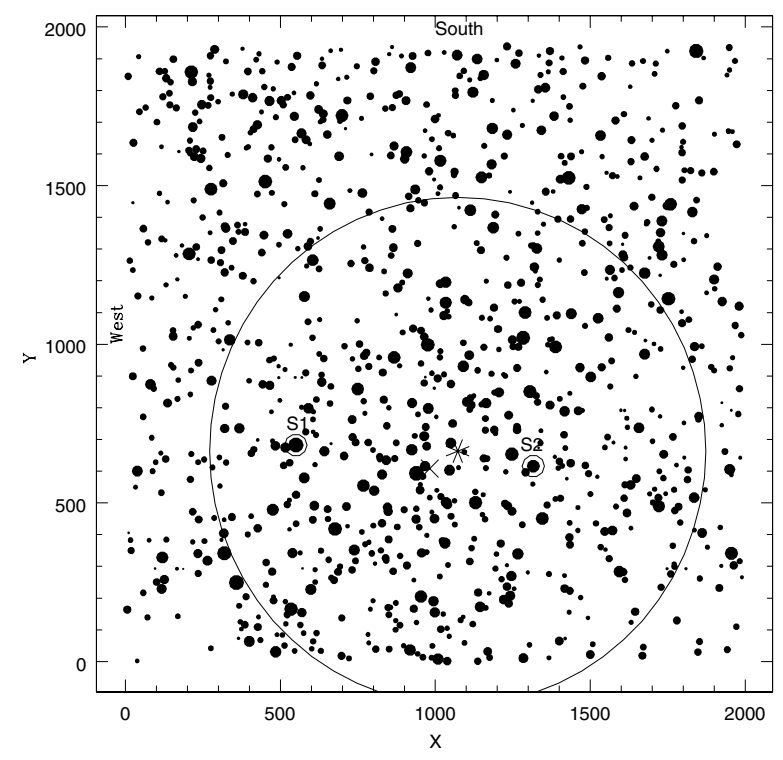

Fig. 1. The observed region of the cluster NGC 146. S1 and S2 denote the stars that show $\mathrm{H}_{\alpha}$ line in emission. The estimated center of the cluster is marked by an asterisk. The radius where increment in the stellar density as seen in the RDP is shown by the large circle.

grism. The image is shown in Fig. 2. The observations were repeated on 25 June, 21 July and 23 November 2004. Low resolution spectra of two stars were obtained on 18 January 2005 in order to study the spectral features in detail. 


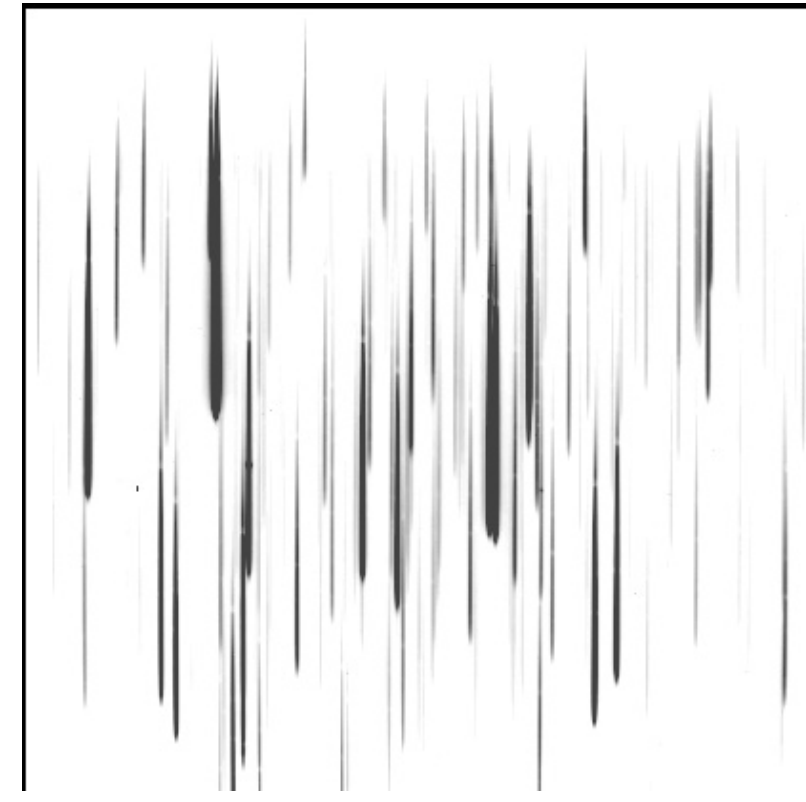

Fig. 2. The slit-less spectra of stars in the region of NGC 146 obtained by using an $R$ filter and a grism in combination. This image is co-added, with a total exposure time of $30 \mathrm{~min}$. The $\mathrm{H}_{\alpha}$ emission in two spectra can be seen $\mathrm{S} 1$ is to the left and $\mathrm{S} 2$ to the right.

\section{Structure of the cluster}

From the cluster plot as shown in Fig. 1, it can be seen that the cluster does not have a well defined core. Hence, the center of the cluster is not well defined by the visual inspection. The cluster center was estimated by taking the average value of the $X$ and $Y$ coordinates of all the stars within a radius of 700 pixels, after initially adopting a visual center. All stars brighter than $V=19$ mag were considered for the center estimation. The estimated center thus found is marked by a cross in Fig. 1. We estimated the radial density profile (RDP) to study the radial structure of the cluster. The stellar density is estimated in radial bins from the center. We computed the RDP for radial bins of 0.5 arcmin. RDPs were estimated for stars brighter than $V=19$ mag and also for stars brighter than 17 mag. The RDP estimated by considering stars brighter than $V=19$ mag was found to have significant scatter, a much better and smoother profile was obtained when stars brighter than 17 mag were considered. The cluster center estimated using only these bright stars is shown with an asterisk in Fig. 1. It can be seen that the two centers are slightly different. The RDP is shown in Fig. 3, by the dots connected by the solid line. It can be seen that within a radius of 4 arcmin, the profile is more or less smooth. Beyond 4 arcmin radius, the profile shows an increament in the stellar density, instead of a decreament. This behaviour is also seen in the profile estimated by considering fainter stars, but is not as prominent as that seen here.

The RDP is fitted with the function $\rho(R) \propto f_{0} /\left(1+\left(R / R_{0}\right)^{2}\right)$, where $R_{0}$ is the core radius at which the density $\rho(R)$ becomes half of the central density, $f_{0}$. It was not able to fit the RDP estimated with stars brighter than $V=19$ mag, which indicates its uneven nature. The best fit to the profile is shown in Fig. 3. Considering that this is the best possible estimate, the core

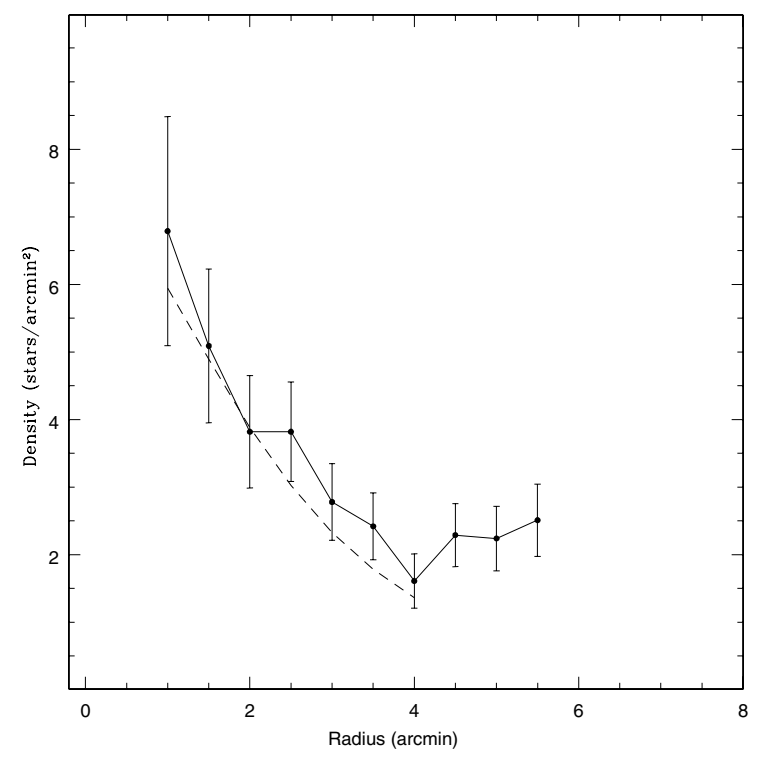

Fig. 3. The radial density of the cluster as a function of the radius (bold line). The dotted line shows the fitted profile.

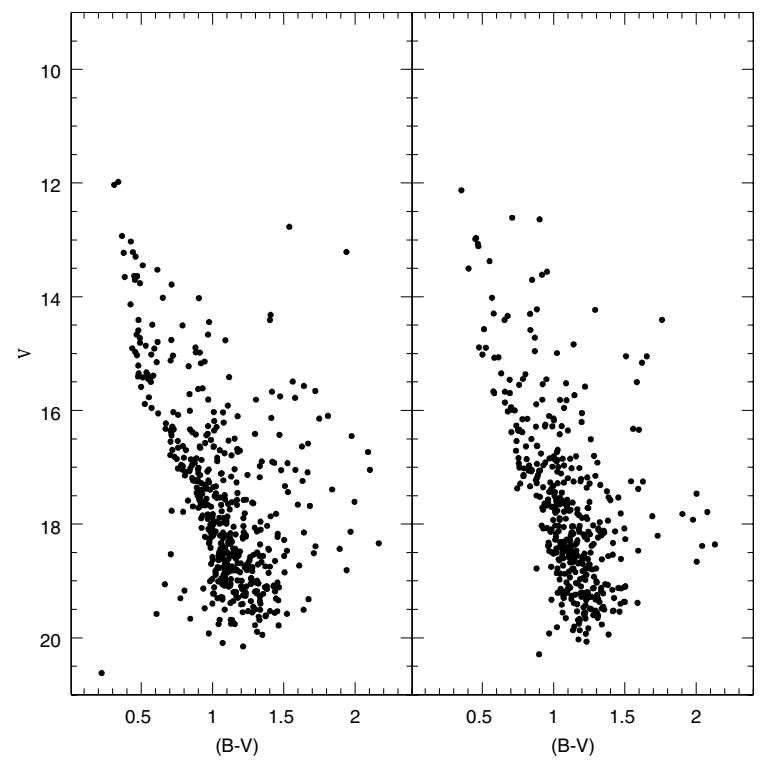

Fig. 4. The $V$ vs. $(B-V)$ CMDs of the stars located in the cluster region (left plot) and the field region (right plot) are shown.

radius of the cluster was estimated as 2.4 arcmin. The fitted profile as shown in the figure also indicated that the density profile beyond the radius of 4 arcmin shows a significant rise. The radius at which this is observed is shown as big circle in Fig. 1. A clear large decrease in the density of bright stars towards the cluster radius can be observed along with the presence of bright stars just beyond the circle. This may indicate a large population of bright stars in the field of the cluster, likely to be caused by spatially extended star formation.

\subsection{Estimation of differential and average reddening}

The colour-magnitude diagram (CMD) of stars located inside the cluster and field are shown in Fig. 4. The stars located 

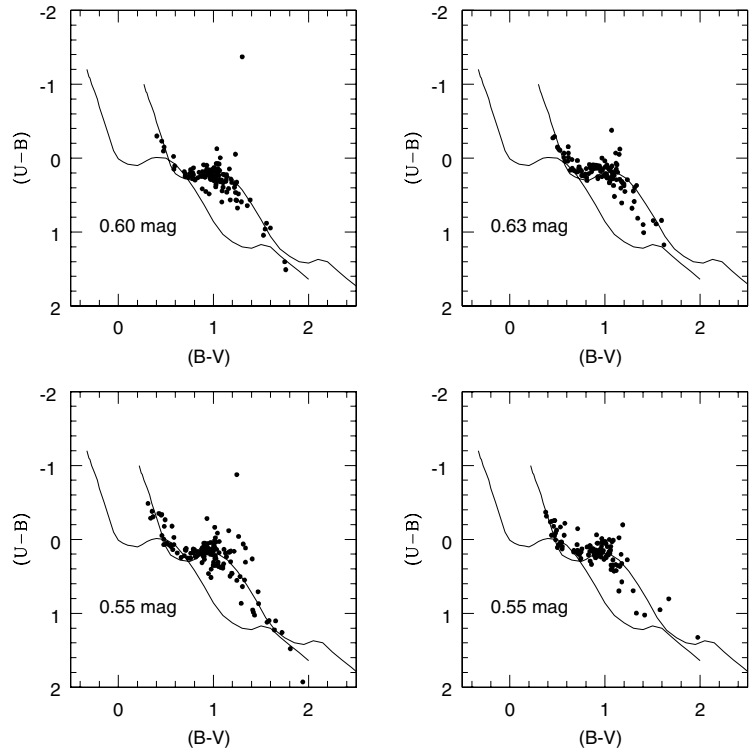

Fig. 5. The ZAMS fit to the $(U-B)$ vs. $(B-V)$ diagrams for the observed regions. The four diagrams represent the four quarters of the observed region. The estimated reddening and distance modulus for each region are indicated.

inside the radius of the cluster are used to make the cluster CMD, whereas stars located outside are used to make the field CMD. The cluster CMD (left panel) shows a well defined main-sequence with stars up to $V=12 \mathrm{mag}$. There is also a population of stars located to the right of the MS. These stars may be field stars or PMS stars, considering the young age of the cluster. The field CMD also shows the presence of a number of bright stars.

The amount of interstellar reddening towards this cluster is estimated using the $(U-B)$ against $(B-V)$ colour-colour diagram (CCDMs). Since the cluster is known to be very young, it is likely that the region of the cluster has variable reddening. The study by Phelps \& Janes (1994) indicated the presence of differential reddening across the cluster. In order to estimate the amount of differential reddening, we divided the observed region into 4 equal parts. The CCDMs for stars within each region were obtained and fitted with the unreddened ZAMS (Schmidt-Kaler 1982). The corresponding CCDMs are shown in Fig. 5, fitted with the ZAMS. The estimated values of $E(B-V)$ are also shown.

It can be seen that there is differential reddening across the cluster with the minimum and the maximum values of reddening being 0.63 and 0.55 mag respectively. The values estimated for each region may also be an average. Further refinement in the estimate is not possible as the number of stars per region becomes too small to reliably estimate reddening. The reddening towards the lower half of the observed region is $0.55 \mathrm{mag}$ and this is where most of the cluster stars are located, as seen from the Fig. 1. The upper half is more reddened and is populated mostly by field stars. Therefore, the mean reddening towards the cluster is estimated to be $E(B-V)=0.55 \pm 0.04 \mathrm{mag}$. The error in the estimation of reddening for each region is $0.02 \mathrm{mag}$ and the average error in the estimation of $(B-V)$ colour is $0.03 \mathrm{mag}$. These errors are quadratically added to obtain the error quoted above. The reddening towards the field region is estimated to be $0.62 \pm 0.04$. Although the reddening values are within the errors, the marginal reduction in the reddening near the cluster region may be due to the material being driven away by the massive stars in the cluster.

\section{Distance to the cluster}

The de-reddened cluster CMD is fitted with the ZAMS to estimate the distance modulus, as shown in Fig. 6. The absolute distance modulus is estimated to be $12.7 \pm 0.2 \mathrm{mag}$. This corresponds to a distance of $3470_{-305}^{+335} \mathrm{pc}$, which can be considered as 3500 pc. Phelps \& Janes (1994) obtained a reddening of $0.70 \mathrm{mag}$ and a distance of $4786 \mathrm{pc}$. Their reddening and distance estimates are much larger than those estimated here. On the other hand, Kimeswenger \& Weinberger (1989) estimated a distance of $3370 \mathrm{pc}$, which is in good agreement with the present estimates.

\section{Estimation of age of the cluster}

The estimation of age of the cluster is done in different ways. First we use the MS turn-off to estimate the age using isochrone fitting as well as synthetic CMDs.

The isochrones from the Padova group, Bertelli et al. (1994), are used to estimate the age. There are a few bright stars present in the cluster, for which we could not estimate the magnitudes, as they were saturated. The magnitudes of these 7 stars are taken from the literature. Magnitudes of 6 stars are taken from Phelps \& Janes (1994) and one from Jasevicius (1964). These stars are used to make the upper MS complete. The isochrone fit to the CMD is shown in Fig. 6. The age of the cluster is estimated from the brightest stars on the MS, since there are no evolved stars. Two isochrones of age 10 and $16 \mathrm{Myr}$ are found to fit the upper MS. Thus we estimate the younger age limit of the cluster as $10 \mathrm{Myr}$, while $16 \mathrm{Myr}$ is also equally probable. The cluster cannot be younger than $10 \mathrm{Myr}$, but it could be slightly older. The upper limit could not be estimated as there are no red giants or super giants. The presence of Be stars in the upper MS indicate that the age of the cluster is similar to the above value, as Be stars are generally seen in clusters in the age range of 12-25 Myr (Meader et al. 1999). Phelps \& Janes (1994) found that the cluster is younger than 10 Myr.

We also estimated the age of the cluster by comparing the features in the observed CMD with synthetic CMDs of different ages. It is seen that the cluster CMD is better understood when compared with a simulated or synthetic CMD made from evolutionary models than with isochrones. A synthetic CMD in general distributes stars along the isochrone corresponding to the age of the cluster, in accordance with the time-scale of each evolutionary state in the CMD. We constructed synthetic CMDs using the theoretical stellar evolutionary models presented by Bressan et al. (1993). We used solar metallicity models to create CMDs. The algorithms used to make synthetic CMDs are presented in Subramaniam \& Sagar (1995) and also used in Subramaniam \& Sagar (1999). We included the effects due to binaries with a mass ratio between $0.75-1.25$ and also the photometric errors in $V$ and $(B-V)$. The Salpeter 

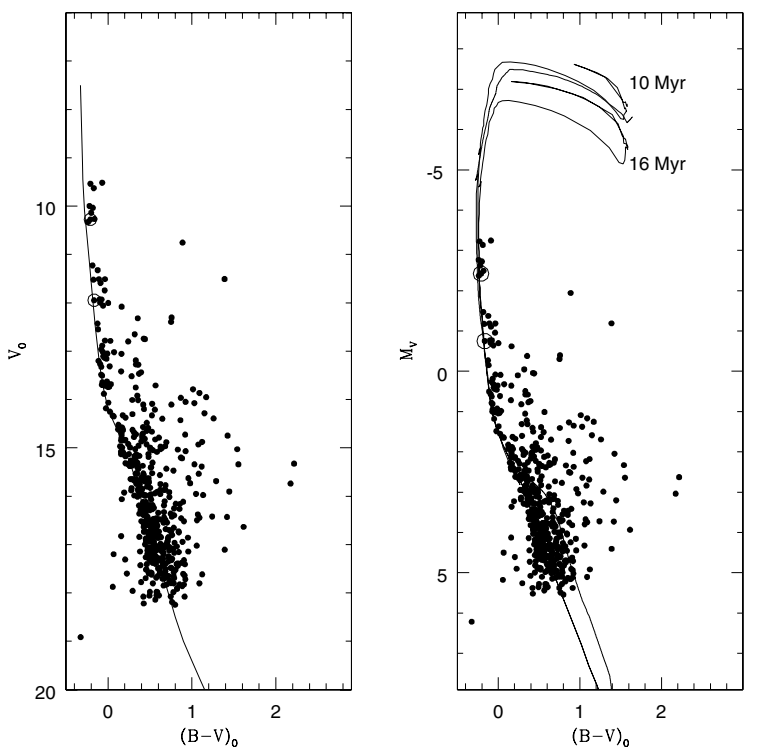

Fig. 6. The ZAMS fit to the unreddened cluster MS in the $V$ vs. $(B-V)$ CMD is shown in the left panel. The extinction corrected magnitudes are plotted on the $y$-axis. The distance modulus is estimated to be $12.7 \mathrm{mag}$. The age of the cluster is estimated by fitting the isochrones to the turn-off of the MS as shown in the right panel. The ages of the fitted isochrones are indicated. The location of two Be stars are shown.

value for the mass function, which is 2.35 (Salpeter 1955), is assumed in general. A control parameter is required to create a synthetic CMD, so that it can be directly compared with the observed CMD. For this, one can either use the number of red giants, or the number of stars near the tip of the MS, below the turn-off. Since there are no red giants in this cluster, we used the stars near the top of the MS for this purpose. In the observed CMD, there are 8 stars between 9.5-10.5 in $V$ magnitude. This is used as the control number. Initially we used a value of $30 \%$ for the fraction of binary stars in the cluster; the resulting CMDs were seen to have too many stars near the evolved part of the CMD, close to the binary isochrone path. The value of the binary fraction is assumed to be zero as even a small value was found to widen the upper MS. Thus the cluster has a very low fraction of binaries, at least among the more massive stars populating the upper MS. Figure 7 shows the observed and the synthetic CMDs for 3 ages, 10, 16 and $20 \mathrm{Myr}$. In the synthetic CMD of age $10 \mathrm{Myr}$, there are stars brighter than the tip of the MS in the observed CMD. In the 16 Myr CMD, the stars in the MS look more or less similar. For ages older than $16 \mathrm{Myr}$, the synthetic CMDs are found to have red giants and their number was found to increase with age. Therefore, the method of synthetic CMDs also constrains the age of the cluster in the range $10-16 \mathrm{Myr}$.

\section{Pre-MS stars and turn-on age}

Since the cluster is found to be young, it is possible that many of the low mass stars are still in the PMS phase. These stars in the CMD occupy the same area as the field stars. Hence, it is not easy to identify a PMS star in a CMD. If PMS stars are present
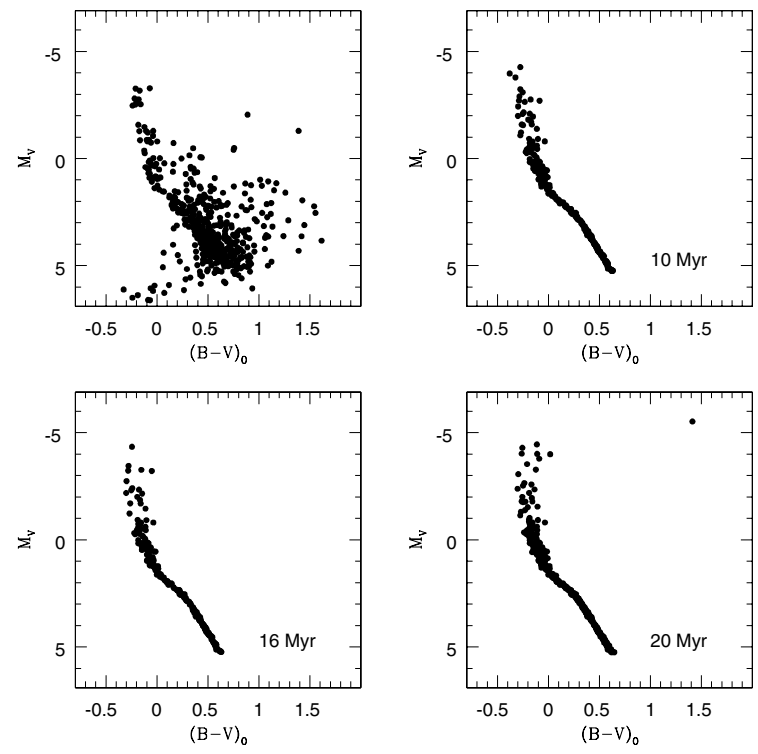

Fig. 7. The observed and the synthetic CMDs for ages 10, 16 and $20 \mathrm{Myr}$.

in the cluster, then these stars can be used to estimate the turnon age of the cluster using PMS isochrones. Therefore, it is important to identify the PMS stars, if they are present. This can be achieved if we identify and remove the field stars present in the region of the cluster. The region outside the circle as shown in Fig. 1 is used for this purpose. This region is considered as the control region to remove the field stars. This region is located very close, just outside the cluster radius. Therefore, it is quite possible that some cluster members, especially low mass stars, are present in the assumed field region. This would result in an over subtraction of low mass stars in the CMD. Since our aim is to identify the presence of PMS stars and not to estimate the statistics of the complete sample, this choice of the control region is justified.

The field stars from the cluster CMD are removed using a technique called zapping technique. This procedure was used in the analysis of LMC clusters by Subramaniam \& Sagar (1995). As the procedure adopted for field star removal is a statistical one, the stars that have the maximum probability to be a field star in the cluster CMD are removed. Therefore the probability of the presence of field stars in the final CMD is very low, though a few field stars still may be present. The field subtracted CMD is presented in Fig. 8 along with the PMS isochrones. The PMS isochrones are taken from Palla \& Stahler (1993). The cluster could have a very young turn-on age of about $0.1 \mathrm{Myr}$ as seen from the figure. There are hardly any stars located on the MS below the turn-on point corresponding to the 3.2 Myr isochrone. Therefore, it is possible that the turn-on age of this cluster is about $3 \mathrm{Myr}$. In order to identify the other known PMS features in the above mentioned stars, we used low resolution spectra of the candidates obtained using the slit-less mode of observations and available $J H K$ data, as described in the following sections. 


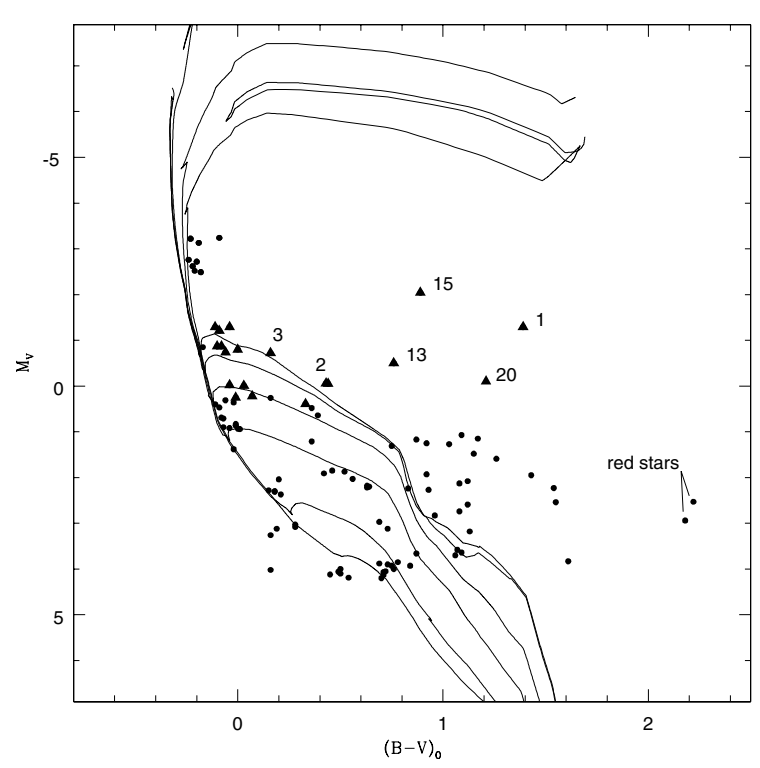

Fig. 8. The age of the cluster as estimated from the MS turn-on and the PMS stars, after correcting for the field stars. The PMS isochrones from Palla \& Stahler (1993) for 0.1, 0.3, 1.0, 3.2, 10, $18 \mathrm{Myr}$ are shown. The triangles show the stars for which spectra are obtained.

\section{Spectra of Be stars and some probable PMS stars}

We obtained slit-less spectra of stars in the cluster to identify emission line stars. This resulted in the identification of two Be stars, whose spectra are shown in Fig. 9. The brighter of the two, S1, shows a few more lines in emission other than $\mathrm{H}_{\alpha}$. The helium lines at $6678 \AA$ and $7065 \AA$ are in emission. Some of the Fe II lines may also be in emission. The above features indicate that hot circumstellar material could be present around S1. The spectral type of S1 is found to be earlier than B3. The other Be star, S2 is fainter and its spectral type is found to be later than B5. In order to study the spectral features of $\mathrm{S} 1$ in detail, we obtained slit spectra of S1 between $3500-9200 \AA$, on 18 January 2005, to confirm the $\mathrm{He}$ and $\mathrm{Fe}$ emission. The flux calibrated spectrum of $\mathrm{S} 1$ is shown in Figs. 10-12. It can be seen that in the blue region, a number of $\mathrm{He} \mathrm{I}$ lines are seen in absorption. The $\mathrm{H} \beta$ line is found to show a P-Cygni profile. In Fig. 11, we identified 5 Fe II lines and 3 He I lines in emission, apart from the $\mathrm{H}_{\alpha}$ emission. The Fe II and $\mathrm{He} \mathrm{I}$ lines are also found to show structures. In the red spectrum, shown in Fig. 12, it can be seen that the O I (8446 $\AA$ ) is in emission whereas the O I (7773 $\AA$, Fig. 11) is in absorption. Another interesting feature is the inverse P-Cygni profiles of the three Ca II lines in Fig. 12. This feature is found to be similar to KK Oph and XY Per (Hamann \& Presson 1992), which are Herbig Ae/Be stars. This is a signature of gas infall. The line profiles are shown in Fig. 13, and were used to estimate the radial velocity of the star and the infall velocity. The structures seen in He I and Fe II may also be due to the self absorption in the line of sight as a result of the infalling gas. As in the case of KK Oph, the velocities were found to be lower in Fe II and higher in Ca II. The radial velocity as estimated from the three Ca II emission profiles is found to be $-120 \pm 25 \mathrm{~km} \mathrm{~s}^{-1}$.

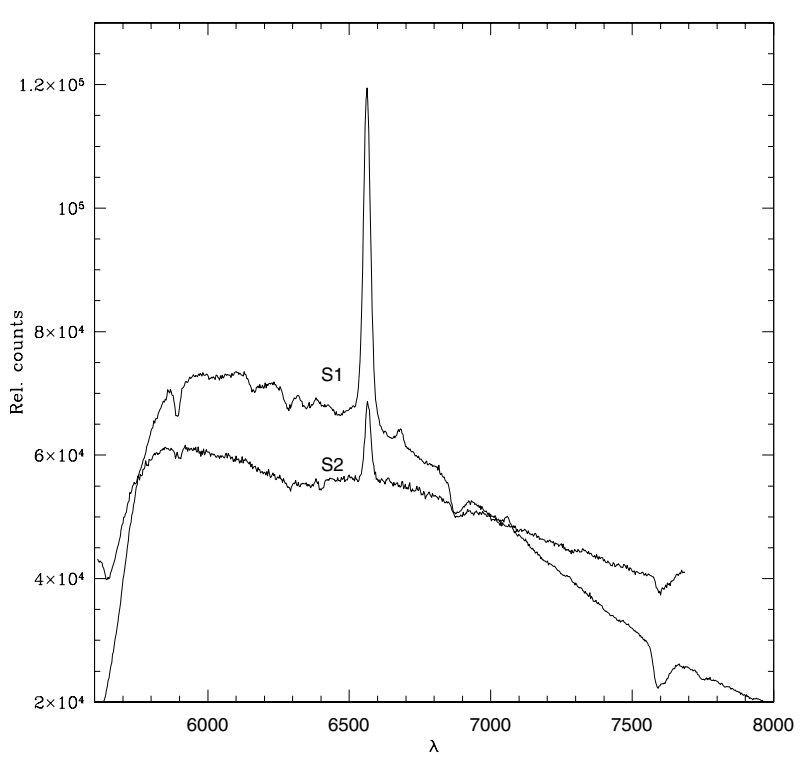

Fig. 9. Spectra of two stars identified as Be stars.

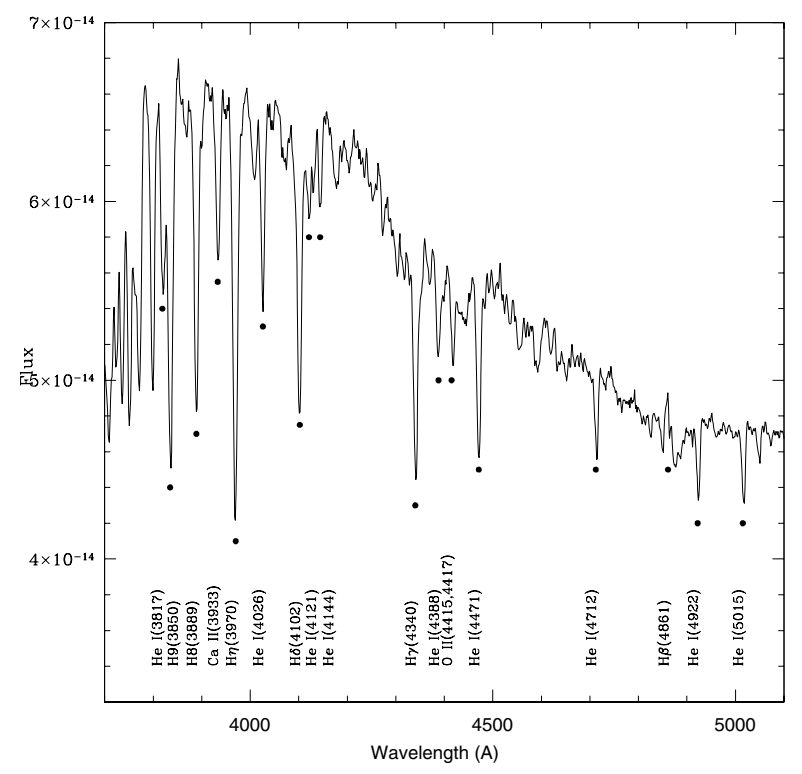

Fig. 10. The blue spectrum of the bright emission star $\mathrm{S} 1$.

The difference in velocity between the emission and the absorption is assumed to be the infall velocity. This was found to be $+300 \mathrm{~km} \mathrm{~s}^{-1}$ for the three Ca II lines and $+150 \mathrm{~km} \mathrm{~s}^{-1}$ for two Fe II and one O I lines.

The slit spectrum of the red star in the CMD denoted as 1 is shown in Fig. 14. This spectrum was also obtained on 18 January 2005. The spectrum indicates that it is a cool star. The strong absorption bands and the absorption feature at $\sim 6500 \AA$ could indicate that the spectral type is similar to an M 2 giant. The absorption feature at $\sim 6500 \AA$ arises from a blend of $\mathrm{Fe} \mathrm{I}, \mathrm{Ca}$ I and Ba II lines and is also seen in FU Orionis type stars (Mundt et al. 1985). The spectrum looks very similar to that of PP $13 \mathrm{~N}$ as shown in Fig. 5, Aspin \& Sandell (2001), except for the $\mathrm{H}_{\alpha}$ emission. They classified PP $13 \mathrm{~N}$ as an M 2.5 giant in the PMS phase. Therefore, it could be a PMS star or a field star. We estimated the 


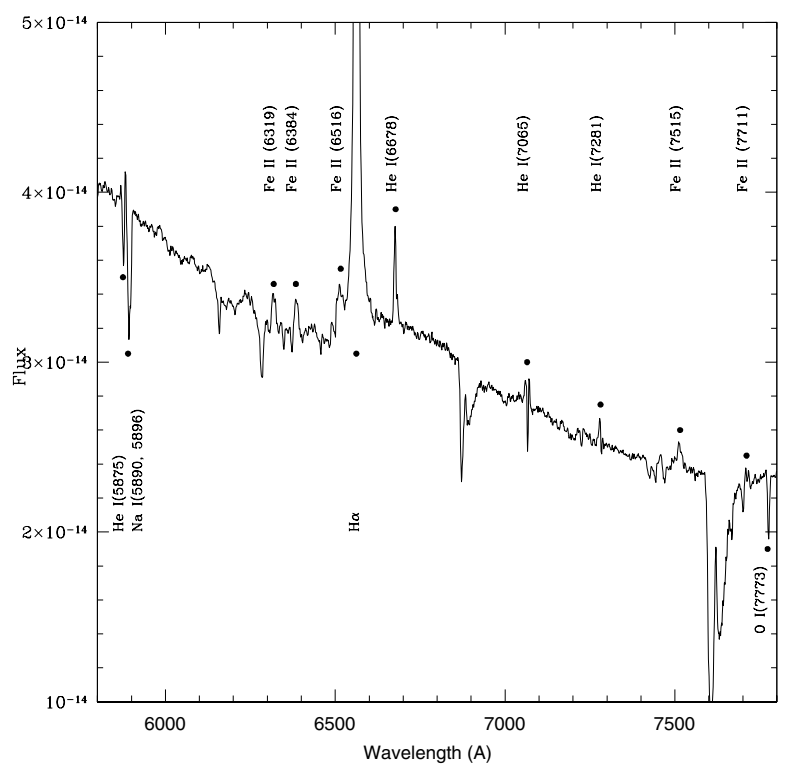

Fig. 11. The spectrum of the bright emission star $\mathrm{S} 1$.

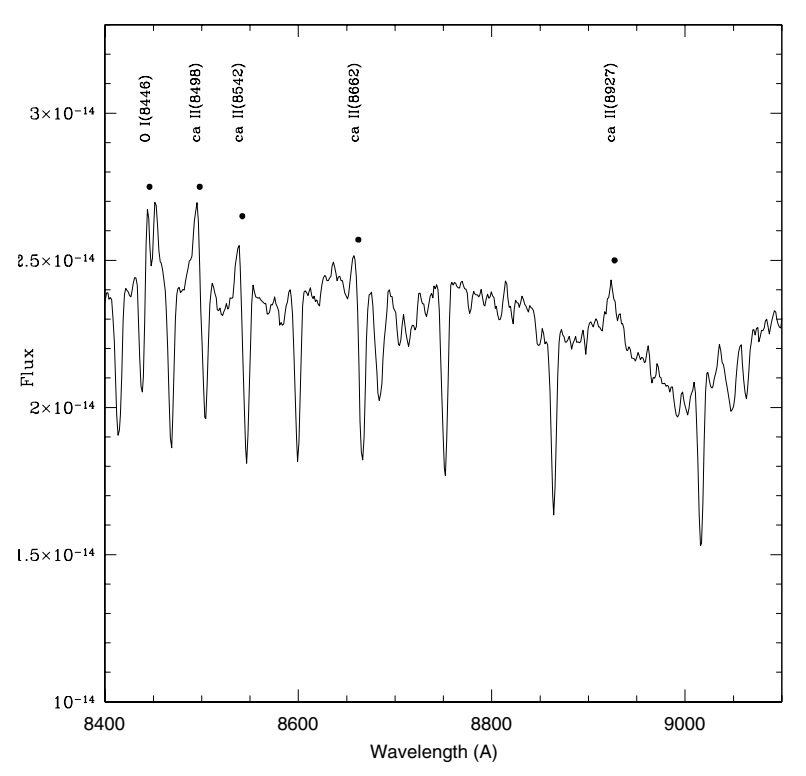

Fig. 12. The red spectrum of the bright emission star $\mathrm{S} 1$.

radial velocity using the three $\mathrm{Ca}$ II lines and it was found to be $-140 \pm 25 \mathrm{~km} \mathrm{~s}^{-1}$. This value is within the errors of $\mathrm{S} 1$. Thus this star is likely to be a cluster member. We searched for fainter stars with $\mathrm{H}_{\alpha}$ in emission in the slit-less spectra, but could not find any. We repeated the observations on three more nights, 25 June 2004, 21 July 2004 and 23 November 2004. None of the stars brighter than $V=15.0$ mag showed $\mathrm{H}_{\alpha}$ emission. We obtained two 15 min exposures on 25 June 2004 and 21 July 2004 and co-added the images to increase the signal. The seeing was poorer on 25 June 2004, which resulted in a lower signal than on 21 July 2004. Therefore, we used the 21 July spectra for further analysis. We extracted the spectra of 18 probable PMS stars shown as triangles in Fig. 8. Spectra of stars brighter than $V \sim 15$ mag had good signal, whereas the spectra of fainter stars had very poor signal. Hence we could only extract spectra of 18 stars brighter than the
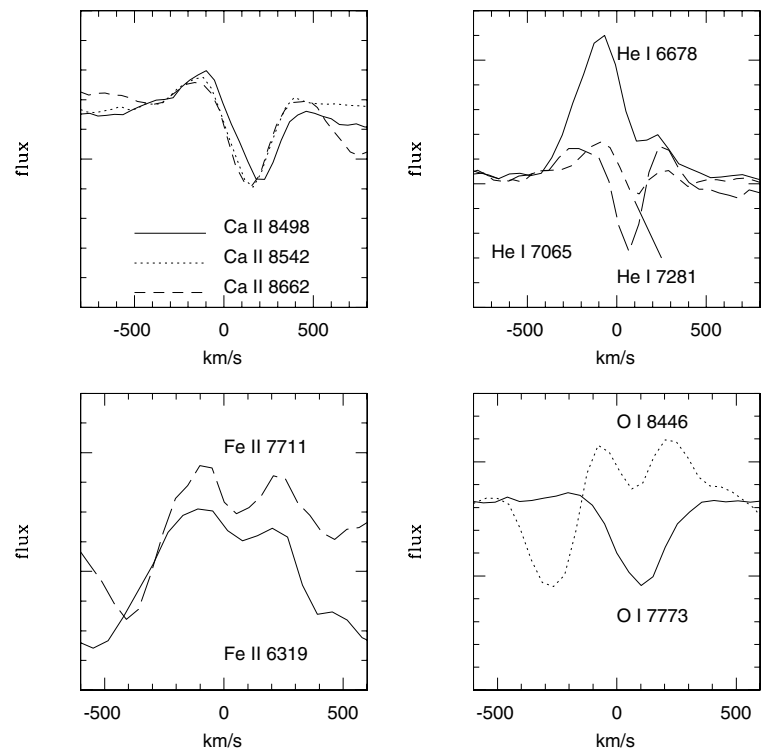

Fig. 13. The line profiles of star S1.

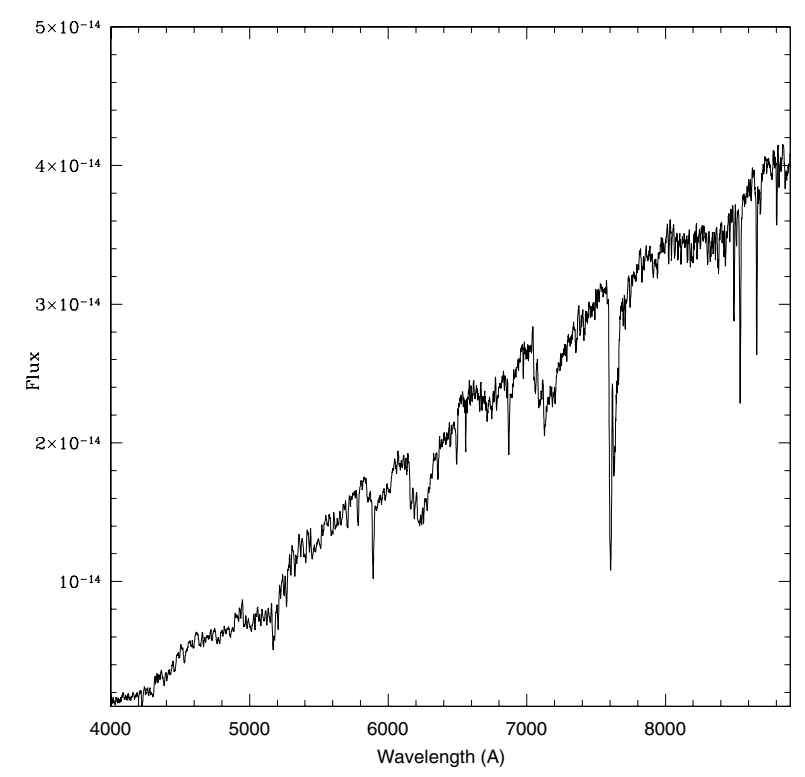

Fig. 14. Spectrum of star 1 .

above limit shown in Figs. 15-17. The magnitudes and location of these stars as in Fig. 1 are given in Table 2. These stars did not show $\mathrm{H}_{\alpha}$ emission in any of the three observations. It can be seen that the spectra are more or less featureless, since the lines are not clearly formed. A noticeable feature is the presence of a very broad $\mathrm{H}_{\alpha}$ line in absorption. The spectra are grouped together and presented such that spectra with deeper $\mathrm{H}_{\alpha}$ are shown in Fig. 15, broader and shallower lined spectra are shown in Figs. 16 and 17. The spectrum of star 15 is shown in Fig. 17, although it does not belong to the group. Spectra of 3 and 5 indicate the likelihood of some emission features. Heske \& Wendker (1985) found that separating stars based on narrow and wide Balmer lines helped to identify PMS stars. They argued that on the way to the MS, the emission lines would slowly disappear in the spectra of PMS stars, with the Balmer lines first. These will switch over to broad 


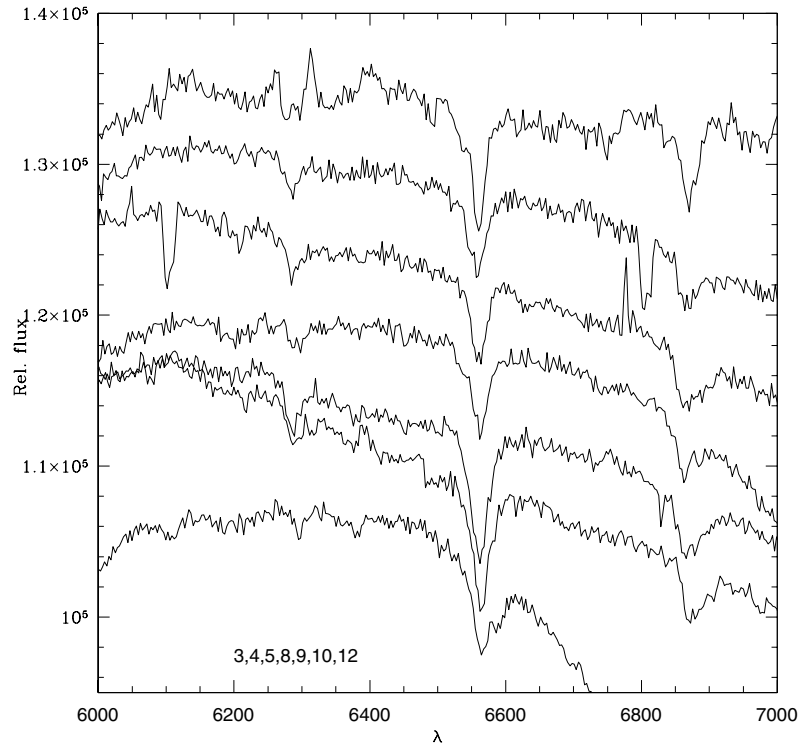

Fig. 15. Spectra of 7 probable PMS stars.

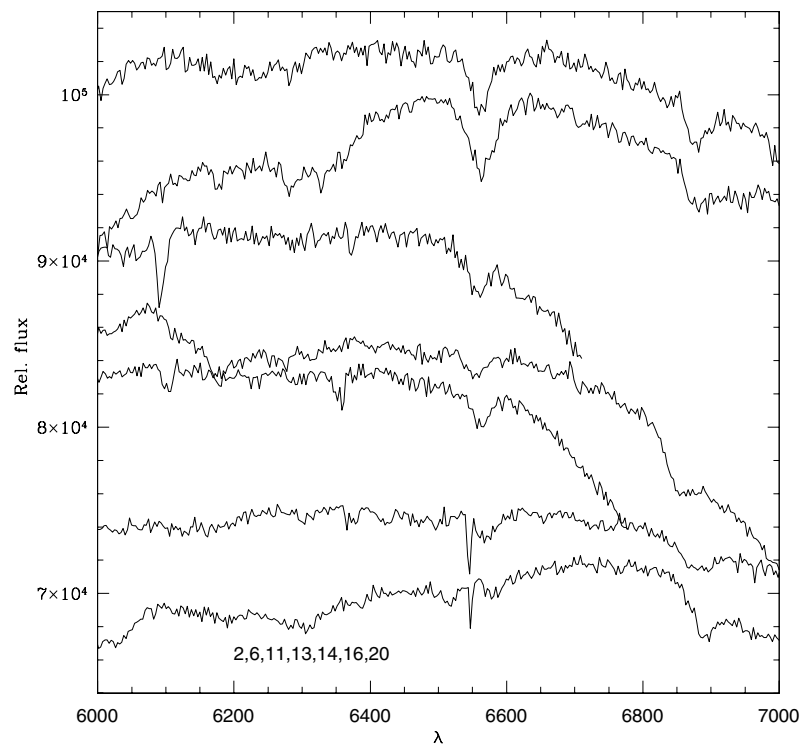

Fig. 16. Spectra of 7 probable PMS stars.

absorption lines and then later become typical Balmer lines. Thus we do not find any clear signature, but only a probable signature of the PMS phase in these stars. In the young open cluster NGC 6611, de Winter et al. (1997) classified stars located redward of the MS as different groups. Following this classification, stars 2 and 3 fall in group II and 1, 13, 15 and 20 fall in group III. Group II is suggested to be an interesting group of likely PMS stars and group III of evolved or field stars or stars with high extinction.

\section{Near infrared colour-colour diagram}

The near infrared (NIR) $J, H, K$ magnitudes of stars in the region of the cluster is available from the 2MASS catalogue. The $K$ vs. $(J-K)$ CMD for stars located within 5 acrmin radius shows that a number of stars have redder colours, probably due to increased extinction. The $(J-H)$ vs. $(H-K)$ colour colour

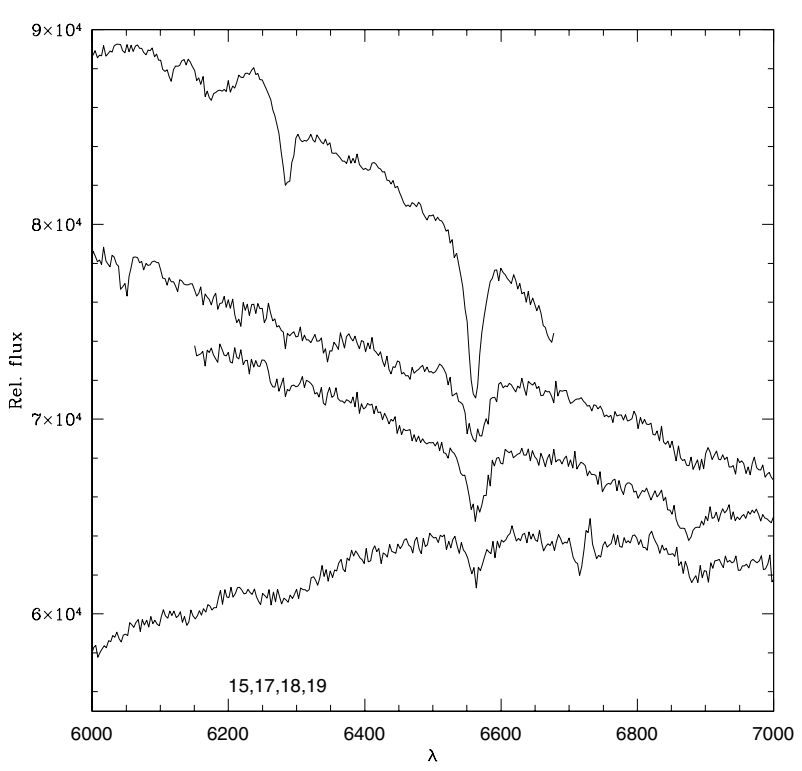

Fig. 17. Spectra of 4 probable PMS stars.

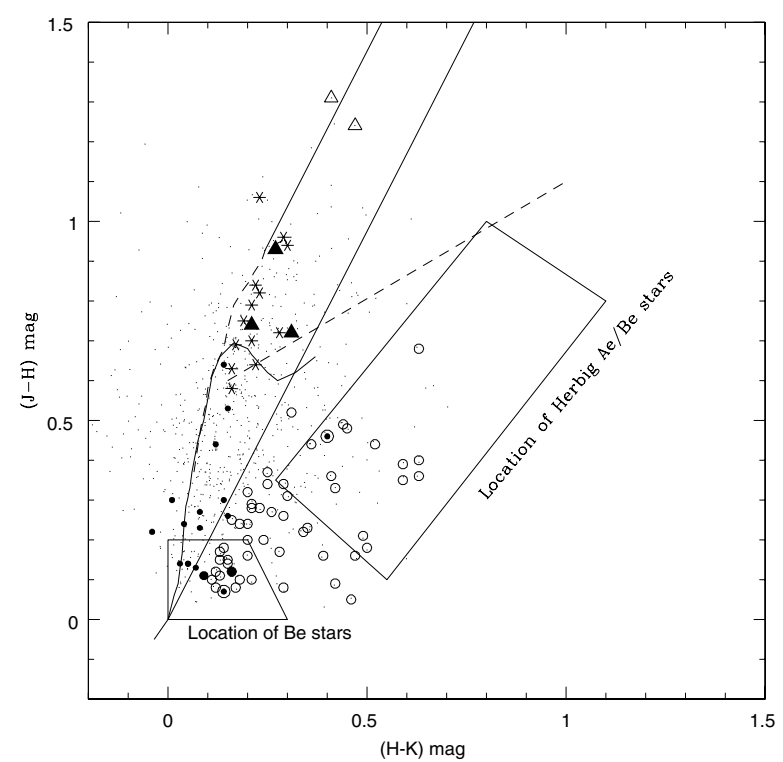

Fig. 18. $(J-H)$ vs. $(H-K)$ colour-colour diagram of stars in the field of NGC 146. The filled circles denote stars for which spectra were obtained. The two $\mathrm{H}_{\alpha}$ emission stars are denoted by dots with a circle around them. The filled triangles are the PMS stars with high extinction. The symbol star denotes objects that are located to the right of the PMS isochrones. The MS and the giant star locations in the CMD are shown (Bessell \& Brett 1988). The location of T-Tauri stars are shown as the dashed straight line (Meyer et al. 1997). The location of Be stars is taken from Dougherty et al. (1994) and the location Herbig Ae/Be stars is taken from Hernandez et al. (2005).

diagram is shown in Fig. 18. A prominent feature seen is the presence of a large number of stars showing NIR excess. These stars are located below the T-Tauri location indicating that these stars are more massive and probably belong to the class of intermediate mass PMS stars. In order to group these stars, we have shown the typical location of Be stars (Dougherty et al. 1994) and Herbig Ae/Be stars (Hernandez et al. 2005). 


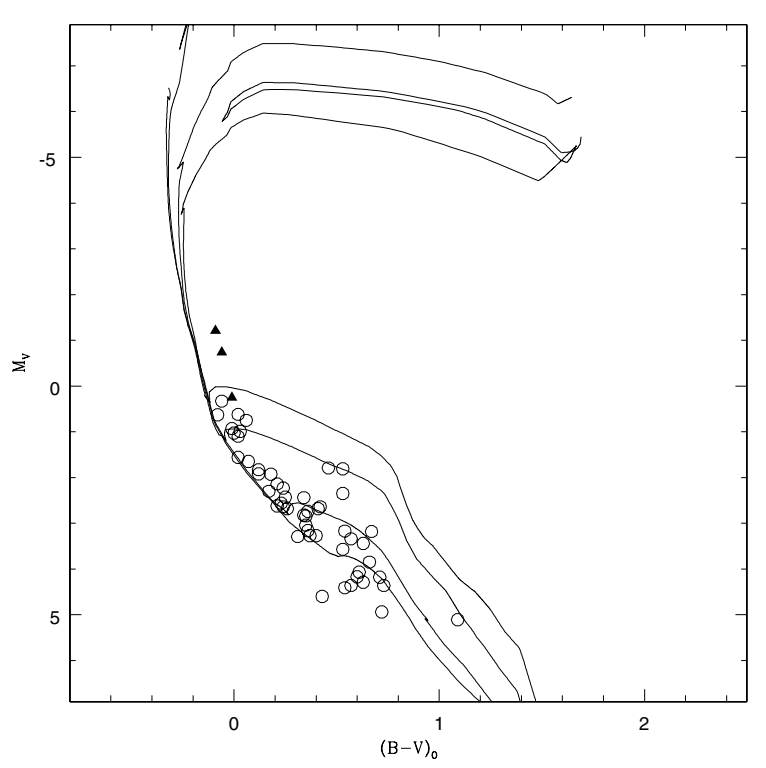

Fig. 19. The optical CMD with stars with NIR excess in the region of the cluster. The PMS isochrones are shown for ages 1, 3.2, 10 and 18 Myr. The symbols have the same meaning as explained in Fig. 18.

The $\mathrm{H}_{\alpha}$ emission star $\mathrm{S} 1$ is found to be located in the Herbig Ae/Be location supporting the evidence obtained from the spectra that it is most likely a Herbig Be star. On the other hand, the star S2 is located in the Be region. The stars which were not found to show $\mathrm{H}_{\alpha}$ emission in their spectra, 4, 5, 9, 10 and 14, were found to be located in the Be region and 4, 9 and 17 have NIR excess. 18 stars are found to have NIR excess and located in the region of Be stars in the colour-colour diagram. The location of Herbig Ae/Be stars is also well populated. We found 17 stars, including $\mathrm{S} 1$, to be located in this region. This may be one of the very few clusters hosting such a large number of intermediate mass PMS stars. We also notice the presence of a large number of stars located between the $\mathrm{Be}$ and the Herbig Ae/Be location. It will be very interesting to determine the nature of these stars. On the whole, inside the cluster radius, we have found 54 stars showing NIR excess. Three stars, 1, 15 and 20 (filled triangles) are found to be located in a region indicating high extinction. In the field subtracted CMD, we found the presence of stars which are located to the right of the PMS isochrones. These stars (star symbol) are found to have very high extinction. The two red stars in the optical CMD are shown as open triangles, which show the highest extinction. Thus a number of stars are found to have high extinction. No optical counterpart could be found for a number of stars identified in the NIR. This is a clear indication of the presence of embedded stars. Thus, NGC 146 shows the presence of a large number of probable intermediate mass PMS stars, embedded stars and stars with high extinction.

The optical $M_{V}$ vs. $(B-V)_{0}$ CMD of the cluster is shown in Fig. 21, where the stars with NIR excess within the cluster region are presented. The stars are referred to by the same symbols as in the earlier diagrams. Some of these stars are not present in Fig. 8, since they may have been identified as field stars. In this figure, we try to identify the population of PMS stars with NIR excess located inside the cluster radius.
Therefore, we have not attempted any field star removal. It can be seen that stars with NIR excess are more or less absent above the 1 Myr isochrone. Most of these stars (open circles) belong to spectral types later than A0. The number is found to increase near the turn on point of the 3.2 Myr isochrone. Therefore, it is very likely that the turn-on age is $\sim 3 \mathrm{Myr}$. This is similar to the value obtained from the field star subtracted optical CMD (Fig. 8). It is also clear that a large fraction of stars located in the cluster region is very young.

\section{Clustering around the Herbig Ae/Be star}

Multi wavelength studies of the environments of several Herbig Ae/Be stars in the optical, NIR and millimeter (Barsony et al. 1991; Aspin \& Barsony 1994; Palla et al. 1995) have shown that young clusters are still partially embedded in the parent molecular clouds and that NIR observations, especially in $K$-band, are best suited to detect the less massive companions of the Herbig Ae/Be star itself. Hillenbrand et al. (1992) found evidence for a correlation between the mass of the Herbig Ae/Be star and the surface density of $K$-band stars detected around it. Testi et al. (1997) confirmed these results. Testi et al. (1999) found that early Herbig Be stars are surrounded by dense clusters of low mass companions. In the present study, where we have found a new Herbig Be star, it will be useful to know whether there is any local stellar density enhancement in its vicinity. We looked at the immediate surrounding of the stars S1 and also some of the bright candidate PMS stars for stellar density enhancement around them. We compared the optical $V$ band data with the 2MASS $K$ band data as shown in Fig. 20. Stellar density enhancement is found near the stars S1, 1 and 20. Mild enhancements were found near the stars 12 and 17 . The star S1 is surrounded by 5 stars that are visible in $K$-band and not in $V$-band. In the case of star 1 , the stellar density is enhanced both in the optical and $K$-band. Around star 20, four stars that are present in $K$-band do not have any counterpart in $V$-band. In this cluster, the densest stellar region is found between the stars S1 and S2. The stellar density to the left of $\mathrm{S} 1$ and to the right of $\mathrm{S} 2$ is less than that between them. Some of the probable PMS stars of lower mass were located close to the higher mass stars. These are indicated in Table 2. That is, two stars each were found to be located close to $\mathrm{S} 1$ and 9 whereas one star each is found to be located close to $\mathrm{S} 1$ and 12.

\section{Discussion}

The cluster is found to have an older turn-off (10-16 Myr) and a younger turn-on age ( $\sim 3 \mathrm{Myr})$. The majority of the stars within the cluster radius is likely to belong to the younger population as indicated by the large number of candidate intermediate age PMS stars (54 stars). This region might have experienced an earlier epoch of star formation between 10-16 Myr and another recent one, when the majority of the stars were formed. A similar case is found in the cluster NGC 6611 (Sagar \& Joshi 1979; Hillenbrand et al. 1993). In this cluster, at least one star is found to be as old as $6 \mathrm{Myr}$ and the age of the PMS population is $\leq 1$ Myr. In the case of NGC 4755, the massive stars 

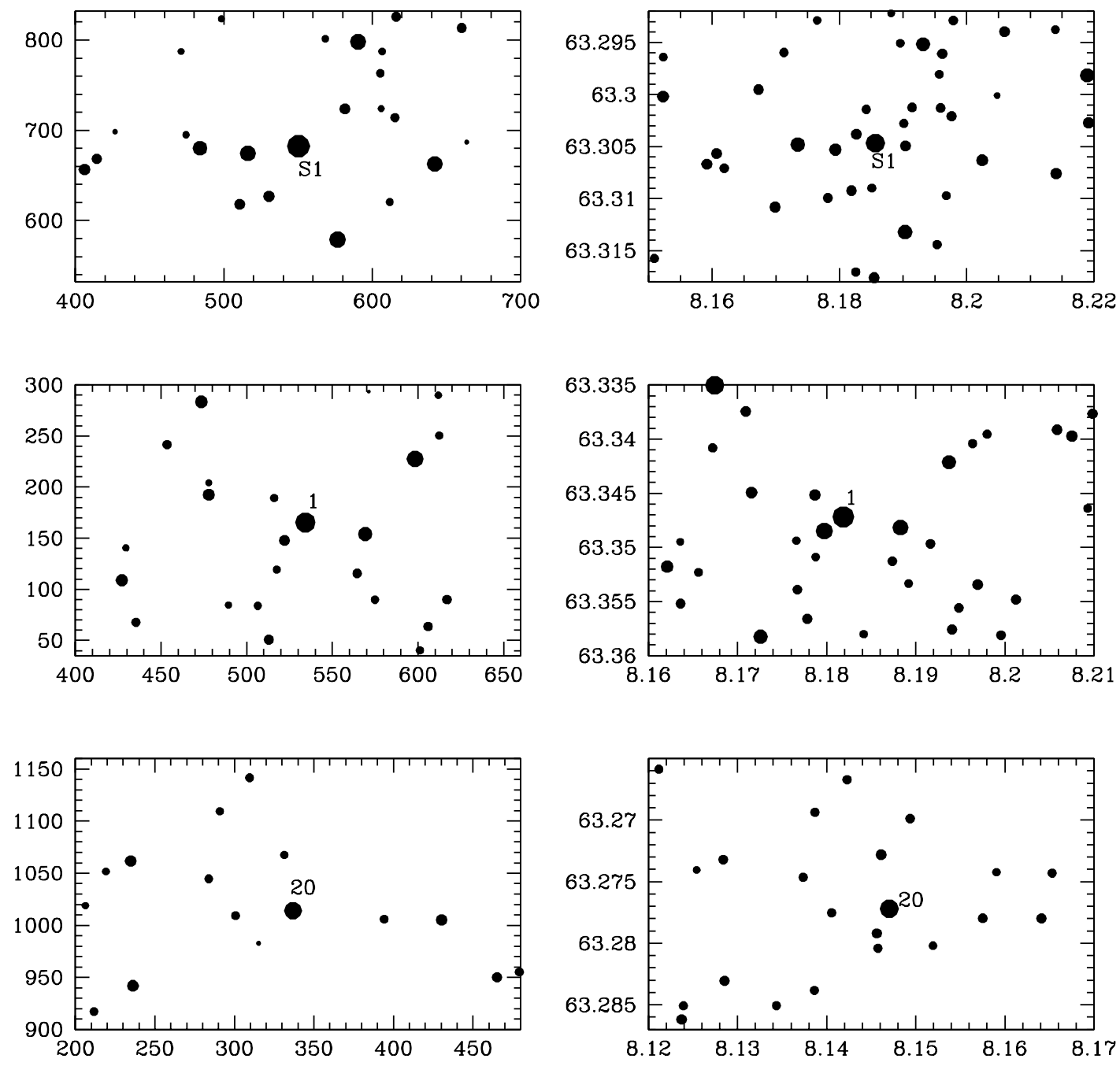

Fig. 20. The $V$ passband (left panels) and the $K$ passband (right panels) are shown around the stars $\mathrm{S} 1,1$ and 20 . The $K$ band data is from 2MASS. The axes are in pixels ( 1 pixel $=0.297$ arcsec) for the $V$ band and in RA (J2000) \& Dec (J2000) for the $K$ band. A local enhancement in the stellar density can be noticed in $K$ band images around these stars.

in the cluster are found to be at least 4 Myr older than the bulk of the low mass stars (Sagar \& Cannon 1995). It is possible that the star formation continued in these clusters for at least 6-7 Myr. It is also likely that multiple epochs of star formation are present in NGC 146.

The low resolution spectra have helped in identifying a few high mass (B type) stars that do not show any emission features, but have infrared excess similar to the Be stars. These stars were characterised by broad $\mathrm{H}_{\alpha}$ absorption lines and are located in the region of Be stars in the colour-colour diagram. Manoj et al. (2002) found three similar stars located in the Orion nebula cluster, which have similar NIR excess but do not show any emission lines in their spectra. This might be due to the fact that the early B-type stars lose their disks and most of the circumstellar material very fast so that the emission features are short lived (Hillenbrand et al. 1993). Delgado et al. (1999) did not find any $\mathrm{H}_{\alpha}$ emission in their candidate PMS stars in IC 4996, which is found to be $~ 8$ Myr old. In the case of NGC 146, there is only one star, S1 which is not only found to retain material around it but also found to show signatures of accretion as well as clustering.
It will be interesting to find out the extent of star formation near this cluster. A study of a larger region around this cluster in optical and NIR is ideal to reveal the star formation history in the Perseus arm around this cluster. Another young cluster, King 14, is found to be located close to NGC 146 and these are one of the candidate double open cluster pairs of Subramaniam et al. (1995). The slit-less spectral observations of King 14 revealed the presence of 1 star showing $\mathrm{H}_{\alpha}$ in emission, which confirms its young nature. If they are located at the same distance, the lateral separation between these clusters is less than $9 \mathrm{pc}$. It is important to study the region containing both these clusters in order to find out if these clusters are binaries. There is no other star cluster located very close to NGC 146, although young clusters like Markarian 50 (Baume et al. 2004) and NGC 7510 (Sagar \& Griffiths 1998; Barbon $\&$ Hassan 1996) are located in the Perseus arm and are similarly old. These clusters are located at $l \sim 110^{\circ}$ and are similarly distant and hence the lateral separation between NGC 146 and the two clusters is about $500 \mathrm{pc}$. We have observed the above two clusters in the slit-less mode to search for emission line stars and we have found that NGC 7510 hosts 2 and 
Table 2. The location and magnitudes of two Be stars and 19 probable PMS stars for which spectra are shown. The $J, H$ and $K$ magnitudes are taken from 2MASS catalogue. The type of star, presence of NIR excess, presence of clustering around the star and the number of probable Herbig $\mathrm{Ae} / \mathrm{Be}$ stars located close to the star are indicated.

\begin{tabular}{ccccccccl}
\hline \hline Number & $X$ & $Y$ & $V$ & $(B-V)$ & $J$ & $H$ & $K$ & Comments \\
\hline S1 & 550.33 & 682.40 & 11.98 & 0.34 & 10.52 & 10.06 & 9.66 & Probable Herbig Be star, clustering, 2 stars \\
S2 & 1316.10 & 616.00 & 13.65 & 0.39 & 12.72 & 12.65 & 12.51 & Be star, 1 star \\
1 & 534.26 & 165.35 & 13.21 & 1.94 & 9.36 & 8.43 & 8.16 & probable PMS star, large $A_{\mathrm{v}}$ clustering \\
2 & 918.10 & 36.42 & 14.45 & 0.98 & 12.16 & 11.63 & 11.48 & group II star, clustering \\
3 & 953.52 & 204.68 & 13.79 & 0.71 & 12.27 & 12.01 & 11.86 & group II star \\
4 & 749.51 & 858.96 & 13.76 & 0.49 & 12.63 & 12.50 & 12.43 & NIR excess \\
5 & 866.91 & 958.25 & 13.64 & 0.45 & 12.61 & 12.47 & 12.44 & \\
6 & 1090.01 & 930.72 & 14.49 & 0.58 & 13.12 & 12.88 & 12.84 & \\
8 & 1387.88 & 991.64 & 13.64 & 0.47 & 12.55 & 12.41 & 12.36 & \\
9 & 1304.90 & 851.16 & 13.30 & 0.46 & 12.21 & 12.10 & 12.01 & NIR excess, clustering, 2 stars \\
10 & 1247.02 & 653.53 & 13.22 & 0.44 & 12.24 & 12.10 & 12.05 & \\
11 & 1289.43 & 1100.30 & 13.53 & 0.61 & 12.11 & 11.88 & 11.80 & \\
12 & 1033.60 & 1130.94 & 14.02 & 0.65 & 12.44 & 12.24 & 12.13 & mild clustering, 1 star \\
13 & 1113.08 & 1422.52 & 14.32 & 1.41 & 11.72 & 11.08 & 10.94 & \\
14 & 1438.33 & 1096.30 & 14.80 & 0.62 & 13.32 & 13.10 & 13.14 & \\
15 & 1283.24 & 1020.19 & 12.77 & 1.54 & 9.66 & 8.92 & 8.71 & large $A_{\mathrm{v}}$ \\
16 & 1590.90 & 1162.93 & 14.77 & 1.09 & 12.64 & 12.20 & 12.08 & \\
17 & 577.48 & 1150.43 & 14.91 & 0.59 & 13.49 & 13.37 & 13.21 & IR excess, mild clustering \\
18 & 1525.15 & 1081.88 & 15.04 & 0.72 & 13.33 & 13.06 & 12.98 & \\
19 & 598.31 & 227.53 & 14.90 & 0.88 & 13.1 & 12.8 & 12.66 & \\
20 & 336.86 & 1013.99 & 14.41 & 1.76 & 11.04 & 10.32 & 10.10 & clustering, large $A_{\mathrm{v}}$ \\
\hline
\end{tabular}

Markarian 50 hosts one star showing $\mathrm{H}_{\alpha}$ in emission. The clusters NGC 663 and NGC 7419, which are known to host a large number of Be stars are also located in this direction, but about $1 \mathrm{kpc}$ closer. Similarly the only known binary cluster pair NGC 869 and NGC 884, which also hosts a number of Be stars, is located in the Perseus arm. Now NGC 146 is found to host a large number of probable $\mathrm{Be}$ as well as Herbig $\mathrm{Ae} / \mathrm{Be}$ stars. The question that comes to mind is whether there is something special about the region forming clusters with a large number of Be stars. It will be interesting to look into this aspect.

\section{Results}

The young cluster NGC 146 is studied using $U B V$ photometry and low resolution spectra to estimate the cluster parameters. The cluster is found to have an older turn-off $(10-16 \mathrm{Myr})$ and a younger turn-on age ( $\sim 3 \mathrm{Myr})$. The difference between them is $\sim 7$ Myr. A significant fraction of stars in the cluster region are found to belong to the younger age. The cluster is located at 3500 pc. Kimeswenger \& Weinberger (1989) presented optical evidence of a spiral arm beyond the Perseus arm, in the second galactic quadrant. NGC 146 was suggested to be part of this spiral arm, with a distance of 4800 pc (Phelps \& Janes 1994). The present distance estimate shows that the cluster NGC 146 is located in the Perseus spiral arm itself as inferred from Fig. 1a of Kimeswenger \& Weinberger (1989). Thus NGC 146 is a cluster located in the Perseus spiral arm of the Galaxy. The NIR colour-colour diagram using the 2MASS JHK photometry of stars in the cluster region showed the presence of a large number of stars (54 stars) with NIR excess, stars with large extinction and embedded stars indicating the youth of the cluster. This cluster is likely to have undergone either continued star formation for $7 \mathrm{Myr}$ or multiple epochs of star formation.

Two Be stars (one Be star and one Herbig Be star) are identified in the cluster for the first time. The Herbig Be star is found to be in the B0-B3 spectral range and the other is found to be later than $\mathrm{B} 5$. We estimated the fraction of Be stars in the B0-B3 spectral range. There is one Be star out of $8 \mathrm{~B}$ stars in the above range, after the removal of field stars. The fraction of Be stars is thus found to be $12.5 \%$. This is similar to the fraction of Be stars found in NGC 581 (Maeder 1999). Also, NGC 581 is found to have an age of $16 \mathrm{Myr}$, very similar to the age of NGC 146. In this study we found that the PMS stars in the B spectral type did not show any $\mathrm{H}_{\alpha}$ emission. They were characterised by broad $\mathrm{H}_{\alpha}$ absorption lines. Thus, the PMS stars in this cluster, that are probably $\sim 3 \mathrm{Myr}$ old do not show any emission features. Thus these stars do not posses any material around them that is left over from the parent molecular cloud.

In this study we demonstrated that the low-resolution spectra of stars in the vicinity of a cluster can be very useful to supplement the results obtained from photometry. We saved a lot of telescope time by using the method of slit-less spectroscopy, instead of obtaining spectra of individual stars. This is a very effective method to study some of the characteristics of stars in young clusters. Here, we used this method to identify the presence of Be stars and confirm the photometrically identified PMS stars. This is the first time we used this method to identify PMS stars in a cluster; we plan to extend this technique to more young clusters. 
Acknowledgements. We thank the referee for encouraging comments. A.S. thanks A. E. Piskunov, Eswar Reddy and Maheswar for helpful discussions. This publication makes use of data products from the Two Micron All Sky Survey, which is a joint project of the University of Massachusetts and the Infrared Processing and Analysis Center/California Institute of Technology, funded by the National Aeronautics and Space Administration and the National Science Foundation.

\section{References}

Aspin, C., \& Barsony, M. 1994, A\&A, 288, 849

Aspin, C., \& Sandell, G. 2001, MNRAS, 328, 751

Barbon, R., \& Hassan, S. M. 1996, A\&AS, 115, 325

Barsony, M., Schombert, J. M., \& Kis-Halas, K. 1991, ApJ, 379, 221

Baume, G., Vazquez, R. A., \& Carraro, G. 2004, MNRAS, 355, 475

Bertelli, G., Bressan, A., Chiosi, C., Fagotto, F., \& Nasi, E. 1994, A\&AS, 106, 275

Bessell, M. S., \& Brett, J. M., 1988, PASP, 100, 1134

Bressan, A., Fagotto, F., Bertelli, G., \& Chiosi, C. 1993, A\&AS, 100, 647

de Winter, D., Koulis, C., The, P. S., et al. 1997, A\&AS, 121, 223

Delgado, A. J., Miranda, L. F., \& Alfaro, E. J. 1999, AJ, 118, 1759

Dougherty, S. M., Waters, L. B. F. M., Burki, G., et al. 1994, A\&A, 290, 609

Hamann, F., \& Persson, S. E. 1992, ApJS, 82, 285

Hardorp, J. 1960, Astron. Abh. Hamb. Sternw. V, No. 7

Hernandez, J., Calvet, N., Hartmann, L., et al. 2005, AJ, 129, 856
Heske, A., \& Wendker, H. J. 1985, A\&A, 151, 309

Hillenbrand, L. A., Massey, P., Strom, S. E., \& Merrill, M. K. 1993, AJ, 106, 1906

Hillenbrand, L. A., Strom, S. E., Vrba, F. J., \& Keene, J. 1992, ApJ, 397,613

Jasevicius, V. 1964, Bull. Vilnius Astron. Obs., 13, 1

Kimeswenger, S., \& Weinberger, R. 1989, A\&A, 209, 51

Maeder, A., Grebel, E. K., \& Mermilliod, J.-C. 1999, A\&A, 346, 459

Manoj, P., Maheswar, G., \& Bhatt, H. C. 2002, MNRAS, 334, 419

Meyer, M., Calvet, N., \& Hillenbrand, L. A. 1997, AJ, 114, 288

Mundt, R., Stocke, J., Strom, S., Strom, K. M., \& Anderson, E. R. 1985, ApJ, 297, L41

Palla, F., Testi, L., Hunter, T. R., et al. 1995, A\&A, 293, 521

Palla, F., \& Stahler, S. W. 1993, ApJ, 418, 414

Phelps, R. L., \& Janes, K. A. 1994, ApJS, 90, 31

Sagar, R., \& Joshi, U. C. 1979, Ap\&SS, 66, 3

Sagar, R., \& Cannon, R. D. 1995, A\&AS, 111, 75

Sagar, R., \& Griffiths, W. K. 1998, MNRAS, 299, 777

Salpeter, E. E. 1955, ApJ, 121, 161

Schimdt-Kaler 1982, in LB, New Series, Gr IV, Vol. 2b (Springer Verlag)

Subramaniam, A., \& Sagar, R. 1999, AJ, 117, 937

Subramaniam, A., \& Sagar, R. 1995, A\&A, 297, 695

Subramaniam, A., Gorti, U., Sagar, R., \& Bhatt, H. C. 1995, A\&A, 302, 86

Testi, L., Palla, F., \& Natta, A. 1999, A\&A, 342, 515

Testi, L., Palla, F., Prusti, T., Natta, A., \& Maltagliati, S. 1997, A\&A, 320,159 